\title{
Paired-Box Gene 8 (PAX8) and Its Association With Epithelial Carcinomas
}

Khalid Khizer ${ }^{1}$, Jaskamal Padda ${ }^{1}$, Anwar Khedr ${ }^{1}$, Fahriba Tasnim ${ }^{1}$, Ola A. Al-Ewaidat ${ }^{1}$, Vinay Patel ${ }^{1}$, Dina Ismail $^{1}$, Victor Yosef Melt Campos ${ }^{1}$, Gutteridge Jean-Charles ${ }^{1,2}$

1. Internal Medicine, JC Medical Center, Orlando, USA 2. Internal Medicine, AdventHealth Orlando Hospital, Orlando, USA

Corresponding author: Dina Ismail, d.ismail94@gmail.com

\begin{abstract}
Cancer is the second most common culprit of mortality in the United States and epithelial carcinomas are considered as one of the most predominant types of cancer. The association between epithelial cancers and paired-box gene 8 (PAX8) has been studied significantly before. PAX8 belongs to the paired-box gene family, which plays an important role in the organogenesis of different body organ systems, especially the thyroid gland, the renal system, and the Müllerian system. Immunohistochemical staining is being used to detect PAX8 expression in different epithelial cancers and differentiate them from PAX8-negative tumors. In follicular, papillary, and anaplastic thyroid carcinomas, targeting the PAX8/peroxisome proliferatoractivated receptors (PPARs) fusion protein is being considered as a potential mechanism for therapy. Moreover, because of its high expression in primary ovarian cancers, PAX8 is being considered as a target for ovarian cancer treatment as well. More studies are needed to test the possibility of using PAX8 as a possible target for managing endometrial carcinomas. In this article, we review the functions of the PAX8 gene, how its mutations lead to the development of certain epithelial carcinomas, how it can be used as a diagnostic or a prognostic marker, and its potential as a therapeutic target for these cancers.
\end{abstract}

Review began 07/26/2021 Review ended 08/15/2021 Published 08/16/2021

๑) Copyright 2021

Khizer et al. This is an open access article distributed under the terms of the Creative Commons Attribution License CC-BY 4.0., which permits unrestricted use, distribution, and reproduction in any medium, provided the original author and source are credited.
Categories: Genetics, Internal Medicine, Oncology

Keywords: epithelial carcinoma, thyroid carcinoma, renal cell carcinoma, endometrial carcinoma, cervical carcinoma, ovarian carcinoma, thymic epithelial carcinoma, ocular epithelial carcinoma, paired-box gene 8 (pax8), paired-box gene family

\section{Introduction And Background}

Cancer is the second leading cause of death in the United States [1]. In 2021, 1,898,160 new cancer cases and 608,570 cancer-related deaths are estimated to take place within the United States [2]. Most of these neoplasms are of an epithelial origin, which arises from the tissues that line all body cavities [3]. Due to this dreadful condition, it has always been a priority to detect cancer at its earliest phase. Although there are many cancer screening and detection methods, it sometimes gets challenging to identify the primary cancer site once it metastasizes. In this situation, PAX8 immunostaining has become a ray of hope.

PAX8 is a member of the paired-box family of genes that plays a crucial role in the development of the kidney, thyroid gland, and Müllerian organs [4-6]. PAX8 positivity has also been demonstrated in several cases of renal, thyroid, and ovarian tumors [7-12]. Due to its high sensitivity and absence of expression in breast, lung, gastrointestinal, and mesothelial malignancies, PAX8 immunohistochemistry is now routinely used as an adjunctive tool in diagnosing tumors, especially when they become metastatic [10, 12-14].

In this review, we discuss the function and pathophysiology of the PAX8 gene. We also shed some light on the role of PAX8 in the development of various cancers, such as thyroid, renal, ovarian, endometrial, cervical, and ocular, as well as thymic epithelial carcinomas. We will also discuss the significance of PAX8 immunostaining in detecting these cancers.

\section{Review}

\section{Definition and function of PAX8}

Genes of the paired-box family encode transcription factors containing the deoxyribonucleic acid (DNA)binding paired-box domain. They have been further subclassified into four groups (I-IV) based on the presence of an octapeptide region, paired-type homeodomain, or both [6, 15]. These transcription factors play a vital role in organogenesis during embryonic development and prevent functional abnormalities in some cells after birth. The PAX8 gene was mainly found to have a vital role in the thyroid gland and kidney embryogenesis [13]. It regulates the differentiation of renal and thyroid follicular cells and controls thyroid hormone release.

Moreover, it has a critical role in the urogenital system morphogenesis, as well as the organogenesis of the central nervous system, eye, mesonephric (Wolffian), and Müllerian ducts [16]. In addition, it was found to 
be expressed in the excretory system of the kidneys, endocervical epithelial cells, uterine endometrium, ovaries, fallopian tubes, seminal vesicles, epididymis, lymphoid cells, and islet cells of the pancreas [17].

\section{Pathophysiology of PAX8}

Congenital Hypothyroidism

A loss-of-function mutation in the PAX8 gene was found to cause congenital hypothyroidism. These mutations limit the ability of the PAX8 gene to bind to DNA and interact with other transcription factors. This interferes with the thyroid gland's normal development and function during embryonic life, resulting in thyroid dysgenesis and abnormally low thyroid hormone levels beginning at birth [18-19].

Tumors

Although the PAX8 transcription factor is crucial in the survival of the differentiated epithelial cells, its expression level can modify the growth rate of these cells. Therefore, it was found that overexpression of this gene under abnormal conditions is related to the development of different tumor types [20]. The PAX8 gene was found to be overexpressed in different tumor types, including thyroid and renal carcinomas, ovarian epithelial carcinomas, and pancreatic neuroendocrine tumors [21]. Based on this, the PAX8 gene can be used for screening and differential diagnosis of these tumors.

\section{Role of PAX8 in thyroid carcinomas}

Thyroid cancers are usually well-differentiated and associated with less mortality. Papillary thyroid cancers are the most prevalent subtype in general. Hürthle cell cancers (HCCs) and follicular thyroid cancer (FTC) together account for $20 \%$ of all thyroid cancers. The latter thyroid cancer types are more dangerous, more frequently discovered in advanced stages, and less responsive to treatment [22].

Peroxisome proliferator-activated receptors (PPARs) are recognized for their role in insulin sensitization and adipogenesis. PPARs, through influence on gene expression of cell signaling pathways, play an essential role in cell cycle control and carcinogenesis [23]. In a subset of FTCs, PPAR translocation has been discovered to play a role as a thyroid protooncogene in some studies [22, 24]. PAX8/PPAR fusion protein (PPFP) is a fusion protein expressed from a neogene which is made from translocation, and it functions to bring PPAR and PAX8 genes together. PPFP has been seen frequently in FTCs [24]. PPFP's impact on PAX8 function has been assessed in a few studies [22]. PAX8 regulates genes for sodium iodide symporter (NIS), thyroid-stimulating hormone receptor (TSHR), thyroid peroxidase (TPO), and thyroglobulin (Tg) promotors. PPFP's effect on PAX8 seems complex; PPFP downregulates the expression of TSHRs and Tg while it upregulates the expression of NIS and TPO [22].

Anaplastic carcinoma can be difficult to differentiate from sarcoma or squamous cell carcinoma (SCC) due to poor immunohistochemistry and morphology. PAX8 is expressed in normal and neoplastic thyroid cells. A study showed PAX8 expression in $76 \%$ of 34 patients with anaplastic carcinoma [25]. In the same study, all the head and neck squamous cell carcinomas had negative results for PAX8. Thus, PAX8 proved to be an excellent marker for follicular epithelial origin, even poorly differentiated ones, to make the differential diagnosis from head and neck squamous cell carcinoma.

Various studies, using reverse transcription-polymerase chain reaction, fluorescence in situ hybridization, nested polymerase chain reaction, or western blot analysis, have pointed out a higher prevalence of expression of PPFP in FTCs [22]. Precise measurements, however, are dependent on study methods and scale. A 36\% incidence has been noted in FTCs and 11\% rates in follicular adenomas. The PAX8/PPAR rearrangement has only been seen in one case of HCC. The papillary variant of FTCs, along with very few cases of HCC, has also shown PPFP expression [26].

Data suggests PPFP can alter pathways which, in turn, stimulates cell proliferation, leading to tumorigenesis. It has also been shown to alter targets of current therapeutic models through PPAR inhibition or PAX8 expression modulation mechanisms. This is why PPFP can be a target in various thyroid cancer treatments, like follicular, anaplastic, and papillary thyroid cancers [22].

\section{Role of PAX8 in renal cell carcinoma}

PAX8 plays a role in the regulation of renal organogenesis. Additionally, it is highly expressed in renal epithelial cells of the adult kidney. PAX8 was discovered to be an oncogene in renal cell carcinoma (RCC) cells by Bleu et al. [27]. They discovered that the transcription factor produced by this gene stimulates the expression of metabolic genes, such as ferroxidase ceruloplasmin, by binding to distal enhancer sites. Ceruloplasmin expression has been related to PAX8 silencing sensitivity in cell lines. Additionally, an inverse association between survival among RCC cases and expression of ceruloplasmin was established. Moreover, there is decreased proliferation of RCC cell lines because of PAX8 silencing. Further analysis revealed that several genes linked to the cell cycle and metabolic processes are also regulated by PAX8. Due to these facts, PAX8 has the potential to play a crucial role in RCC development [27]. PAX8 can be detected 
in all segments of the renal tubules, from the proximal tubules to the renal papillae as well as in the parietal cells of Bowman's capsule [7]. Several studies have also shown that PAX8 can be detected in samples of different types of RCC which are summarized in Table 1 [7, 13, 28-30].

\begin{tabular}{|c|c|c|c|c|}
\hline & Clear Cell RCC & Papillary RCC & Chromophobe RCC & Renal Oncocytoma \\
\hline Tong et al., 2009 [7] & $98 \%(58 / 59)$ & $90 \%(19 / 21)$ & $82 \%(9 / 11)$ & $95 \%(21 / 22)$ \\
\hline Barr et al., 2015 [28] & $80 \%(165 / 207)$ & $95 \%(39 / 41)$ & $100 \%(6 / 6)$ & NA \\
\hline Hu et al., 2012 [29] & $93 \%(78 / 84)$ & $95 \%(54 / 57)$ & $80 \%(53 / 66)$ & $94 \%(15 / 16)$ \\
\hline Tacha et al., 2011 [30] & $91 \%(86 / 94)$ & $100 \%(14 / 14)$ & $57 \%(4 / 7)$ & NA \\
\hline Laury et al., 2011 [13] & $93 \%(115 / 123)$ & $76 \%(19 / 25)$ & $80 \%(4 / 5)$ & $81 \%(13 / 16)$ \\
\hline \multicolumn{5}{|c|}{ TABLE 1: Summary of the Studies Reporting the Percentage of PAX8 Expression in Renal Ce } \\
\hline NA: not available; RCC: & arcinoma & & & \\
\hline
\end{tabular}

Furthermore, Ozcan et al. reported the frequency of PAX8 expression to be $82 \%$ - $89 \%$ among American patients with RCC [31]. Knoepp et al. also noted the frequency to be $88 \%$ among American patients [32].

Due to the high expression of PAX8 in RCC, it can be used for screening as well as risk stratification [32]. Also, it can be used as a diagnostic tool that can play a supportive role in diagnosing RCC, alongside core biopsy, as it can be challenging to obtain a sample from metastatic lesions. About $5 \%$ of primary tumors are considered unclassified, even after resection, due to poor differentiation or a mixed morphological pattern [28]. Also, metastatic tumors arising from the kidney may resemble other malignancies as they may have different characteristics than the primary tumor or may be poorly differentiated [33]. Thus, it may be challenging to detect tumors in advanced stages. For these reasons, pathologists are inclining more towards the use of immunohistochemistry in diagnosing primary and metastatic RCC to distinguish it from other primary tumors. A survey conducted by the International Society of Urologic Pathologists found that about $90 \%$ of pathologists use immunohistochemistry to confirm the diagnosis of metastatic RCC [34]. While many pathologists reported using various markers, such as cytokeratin 7 (CK7), vimentin, and a cluster of differentiation 10 (CD10), most of them stated PAX2 and PAX8 to be the most valuable markers. However, in another study, PAX8 was found to be more sensitive than PAX2 and considered eligible to play an essential role in the diagnosis of primary renal neoplasm [35].

\section{Role of PAX8 in endometrial carcinoma}

PAX8 has also been associated with certain endometrial cancers due to its role in cell growth. Therefore, a study on 229 patients was done to find the correlation of PAX8 expression in specific clinical parameters of endometrial cancer [36]. Positive PAX8 results were correlated with high tumor grade $(\mathrm{P}=0.002)$, type 2 tumor subtype $(\mathrm{P}<0.0001)$, and lymphovascular invasion $(\mathrm{P}=0.0186)$. In addition, the five-year survival with a disease-free state was calculated as $49.88 \%(\mathrm{P}=0.02028)$ in PAX8-positive patients compared to $72.12 \%$ in PAX8-negative patients. Also, PAX8-negative patients were associated with $74.36 \%$ chances of recurrence-free survival compared to $52.11 \%$ in PAX8-positive.

In another study, 106 patients with primary endometrial carcinoma were studied over a 10-year period, 97 of which were investigated further based on eligibility [37]. P53 and PAX8 expressions were calculated using an immunohistochemical technique and data was clinically correlated. PAX8 was found to be positive in $72.1 \%$ of 97 patients, $72.7 \%$ of 77 endometrial cancer patients, and $72.2 \%$ of 18 patients with non-endometrioid cancers. Histological type, grade, and p53 expression data also showed a positive correlation. Vessel space involvement, lymph node involvement, stage of the tumor, and age were also assessed, with no correlation with PAX8. Patient survival was also shown to have no statistically significant correlation with PAX8 with a five-year survival rate of $76 \%$ in PAX8-positive patients compared to $72 \%$ in PAX8-negative patients.

In yet another study, 52 endometrioid endometrial cancers, 21 human papillomavirus-related endocervical cancers, 21 serous endometrial cancers, 11 benign mesonephric growths, and 58 normal endometrium patients were studied for PAX8 expression [38]. Both serous and endometrioid endometrial cancers were shown to have a high association with serous endometrial cancer, expressing significantly high levels of PAX8. Endocervical adenocarcinomas were also associated with a high frequency of PAX8 expression but not as much as endometrial adenocarcinomas. High PAX8 expression in such various types of intrauterine malignancies makes it less useful as a diagnostic marker. Significantly low expression of PAX8 was noted with the normal or benign proliferation of the endocervix and endometrium patients. However, in 


\section{Cureus}

extrauterine sites, it can be a valuable marker for adenocarcinomas of uterine origin. The sensitivity of identifying metastasis is less and depends upon tumor cells in the metastatic site [38]. In general, PAX8 can be considered a poor prognostic marker in patients with endometrial carcinomas and can be targeted for therapy in selected patients, the utility of which needs more clinical data [36].

\section{Role of PAX8 in cervical carcinoma}

PAX8 is a strictly regulated transcription factor that has also shown increased immunostaining in cervical carcinomas. As PAX8 plays an essential role in the embryogenesis of the Müllerian ducts, PAX8 expression is noted in carcinomas developed from this region. Although the PAX8 gene gives rise to four isoforms through alternative messenger ribonucleic acid (mRNA) splicing, there is no available data that can determine which PAX8 isoform is present in cervical tissues and cervical carcinoma. Instead, numerous previously unreported PAX8 aberrant transcripts were found in both cervical carcinoma-derived cell lines and tumor samples [39].

On a separate note, it has been established that the E2F transcription factor 1 (E2F1) promoter is transcriptionally regulated by PAX8 directly, and RB1 depletion enhances E2F1 transcription leading to persistent cell growth [40]. When a cell acquires TP53 or RB1 mutation, it may generate epithelial lesions, such as endometrial cancer [41]. Similarly, we can detect PAX8 upregulations in cervical cells infected with human papillomavirus (HPV) as viral proteins disrupt TP53 and RB1 functions. In a recent study, RNA analysis in cervical samples uncovered upregulation of PAX8 transcripts in HPV-positive lesions, presumed to be the main culprit behind cervical carcinoma [42]. Several studies reported PAX8 expression in adenocarcinoma, SCC, endometrioid adenocarcinoma, and adenosquamous carcinoma of the uterine cervix $[13,30-31,38,43-51]$.

Shukla et al. reported PAX8 immunostaining in 64 of 66 cases of adenocarcinoma in situ and 11 of 55 cases with high-grade squamous intraepithelial lesions of the cervix [43]. A case report noted that PAX8 immunostaining was valuable in diagnosing metastatic cervical cancer of the breast [44]. Several other studies demonstrating PAX8 immunostaining in cervical cancers are summarized in Table 2 [13, 30-31, 38, 45-51].

\begin{tabular}{|c|c|c|c|c|}
\hline & Adenocarcinoma & SCC & Endometrioid Adenocarcinoma & Adenosquamous Carcinoma \\
\hline Laury et al., 2011 [13] & $1 / 2$ & $2 / 2$ & NA & NA \\
\hline Tacha et al., 2011 [30] & $5 / 6$ & $1 / 60$ & NA & $1 / 3$ \\
\hline Tong et al., 2011 [45] & $0 / 5$ & NA & NA & NA \\
\hline Woodard et al., 2011 [46] & $12 / 19$ & NA & $6 / 9$ & NA \\
\hline Danialan et al., 2013 [47] & $2 / 12$ & NA & NA & NA \\
\hline Yemelyanova et al., 2014 [38] & $18 / 21$ & NA & NA & NA \\
\hline Goyal et al., 2014 [48] & 13/15 & NA & NA & NA \\
\hline Liang et al., 2016 [49] & $35 / 43$ & NA & NA & NA \\
\hline Ozcan et al., 2011 [31] & NA & $0 / 9$ & NA & NA \\
\hline Gailey et al., 2013 [50] & NA & $3 / 11$ & NA & NA \\
\hline Wong et al., 2017 [51] & $14 / 20$ & 8/103 & $5 / 6$ & $2 / 7$ \\
\hline
\end{tabular}

TABLE 2: Summary of the Studies Reporting the Percentage of PAX8 Expression in Cervical Carcinomas

NA: not available; SCC: squamous cell carcinoma

Although several specimens expressed PAX8 immunostaining in cervical cancer samples in several studies, they were not highly specific [13, 30-31, 38, 45-51]. From the aforementioned studies, we can see that most SCC and a significant number of adenocarcinomas of the cervix lacked PAX8 staining. Hence, PAX8 immunostaining can play an essential role as an adjunct tool in diagnosing cervical cancer, but a negative result cannot rule out the diagnosis [51]. Furthermore, the usefulness of PAX8 staining in differentiating endocervical lesions from endometrial lesions is limited [52].

Role of PAX8 in ovarian carcinoma 
Ovarian cancer has the most unfavorable prognosis of all cancers of the female reproductive system. This is due to its subtle presentation and the absence of reliable screening and diagnostic tests which makes early detection of this disease very challenging. The high-grade serous carcinoma subtype is the most prevalent and severe type of ovarian cancer [53]. The PAX8 gene is expressed in the adult fallopian tube epithelial cells but not in the ovarian epithelial cells. However, it was found that PAX8 gene expression was clearly detected in ovarian epithelial cancer cells. This indicates that the origin of these cancer cells is from the fallopian tube epithelial cells [54].

The exact role of the PAX8 gene in ovarian epithelial cell carcinogenesis is still unclear. However, the PAX8 gene was found to play an essential role in the motility, adhesion, invasion, and tumorigenesis of ovarian cancer cells by modulating these cells' interaction with the extracellular matrix. This characterizes the metastatic way of this cancer, which is direct seeding and invasion of the peritoneal cavity [55].

The current use of cancer antigen (CA)-125, estrogen receptor, and progesterone receptor for the diagnosis of ovarian cancer are unreliable due to their low specificity and sensitivity. This further complicates the early detection of this disease [56-57]. Based on the immunohistochemical studies, the PAX8 gene was clearly expressed in the primary ovarian cancer cells but not in the metastatic ovarian cancer cells, which indicates the high specificity of this gene expression in primary ovarian cancer cells. Therefore, the PAX8 gene is being considered as a potential effective diagnostic marker of ovarian cancer. Moreover, it was discovered that the level of this gene expression is proportional to the degree of differentiation of cancer cells. It was found that higher PAX8 gene expression was associated with a lower cancer cell differentiation and lower survival rate. This gives PAX8 gene the advantage of being a prognostic factor of ovarian cancer as well [57].

Due to the enormous diversity of ovarian cancer cell mutations, it has been challenging to find targets for the effective treatment of this disease. However, the PAX8 protein is regarded to be a promising target for ovarian cancer treatment based on its pervasive expression in these cancer cells [54]. This gives hope to decrease this aggressive tumor's burden and progression and further increase the patient's survival.

\section{Role of PAX8 in thymic epithelial carcinoma}

Neuroendocrine cancers of the lung and neuroendocrine tumors from the thymus have similar clinical presentations, although they are very different in their biological behavior. Studies have shown a significantly high level of mortality associated with the atypical origin of neuroendocrine tumors [58-59]. The five-year survival of thymus origin carcinoid tumors was calculated to be $50 \%$ compared to atypical carcinoid tumors having a low rate of $5 \%$. This makes the differential of these two based on origin very important. PAX8 has been drawing attention as a potential marker. PAX8 is expressed in epithelial tumors in different organs, as well as in thymic epithelial neoplasms. According to many studies, pulmonary neuroendocrine cancers have failed to express PAX 8. Thus, PAX8 can be used as an immunohistochemical marker that can be used to differentiate lung from thymic origin tumors [60]. In yet another study, PAX8 immunoreactivity was assessed in 31 thymic carcinomas, 30 patients with World Health Organization (WHO) type B thymoma, and 30 patients with WHO type A thymomas [61]. Seventy-seven percent of thymic cancers, $93 \%$ of WHO type B thymomas, and 100\% of WHO type A thymomas showed positive PAX8 immunoreactivity. In another small study on 13 thymic cancer cases and 15 poorly differentiated lung cancer cases, patients were analyzed for PAX8 expression [62]. In that study, $69.2 \%$ of thymus origin cancers showed PAX8-positive results compared to $5.8 \%$ in lung cancer patients. The study concluded that PAX8 had a high diagnostic value in patients with thymic cancers.

\section{Role of PAX8 in ocular epithelial carcinoma}

Intraocular metastasis of malignant tumors represents the most common intraocular tumor. However, retinoblastoma and malignant uveal melanoma are the most common primary intraocular tumors [63]. The intraocular expression of the PAX8 gene was observed in the retinal neurons, the iris sphincter pupillae muscle, and the dilator muscle complex. It was also found to be expressed in the epithelium of the cornea, lens, iris pigment, and ciliary body. A subset of intraocular tumors demonstrated a clear PAX8 gene expression. This includes epithelial and neuroepithelial ciliary body tumors, retinoblastoma, and uveal melanoma. However, its expression was not detected in all melanocytic and retinal pigment epithelial tumors. Therefore, the PAX8 gene is being considered as a target of an effective diagnostic marker of different intraocular tumors [64-65]. Although PAX8 is expressed in the lens epithelium, it was found to be absent in the lens epithelium with mesenchymal transition, including capsular fibrosis and subcapsular cataract [66]. Based on that, it does not represent a reliable diagnostic marker for these lesions.

\section{Conclusions}

The object of this review is to examine the association between PAX8 and epithelial carcinomas. PPAR translocation and fusion with PAX8 was found to play a role in the pathogenesis of some types of follicular thyroid carcinoma. PAX8 is being evaluated by pathologists using immunohistochemistry to diagnose primary and metastatic RCC. PAX8 is also being used to differentiate primary ovarian carcinoma from metastatic carcinomas and as an additional way to confirm the diagnosis of cervical carcinomas. Moreover, 
PAX8 is utilized to differentiate thymic epithelial neoplasms from other pulmonary and mediastinal epithelial masses. Intraocular ciliary body epithelial neoplasms were found to be PAX8-positive; this is why it is considered a valuable tool to differentiate these tumors from other intraocular melanocytic tumors. In addition, PAX8 expression is regarded as a poor prognostic factor in cervical and endometrial epithelial carcinomas. The role of PAX8 as a treatment target has not been thoroughly studied yet, so more studies are needed to evaluate the potential of targeting PAX8 to treat different PAX8 positive epithelial carcinomas, especially thyroid, ovarian, and endometrial carcinomas.

\section{Additional Information \\ Disclosures}

Conflicts of interest: In compliance with the ICMJE uniform disclosure form, all authors declare the following: Payment/services info: All authors have declared that no financial support was received from any organization for the submitted work. Financial relationships: All authors have declared that they have no financial relationships at present or within the previous three years with any organizations that might have an interest in the submitted work. Other relationships: All authors have declared that there are no other relationships or activities that could appear to have influenced the submitted work.

\section{References}

1. An Update on Cancer Deaths in the United States . (2021). Accessed: June 14, 2021: http://www.cdc.gov/cancer/dcpc/research/update-on-cancer-deaths/index.htm.

2. Siegel RL, Miller KD, Jemal A: Cancer statistics, 2020. CA Cancer J Clin. 2020, 70:7-30. 10.3322/caac.21590

3. Hinck L, Näthke I: Changes in cell and tissue organization in cancer of the breast and colon . Curr Opin Cell Biol. 2014, 26:87-95. 10.1016/j.ceb.2013.11.003

4. Lang D, Powell SK, Plummer RS, Young KP, Ruggeri BA: PAX genes: roles in development, pathophysiology, and cancer. Biochem Pharmacol. 2007, 73:1-14. 10.1016/j.bcp.2006.06.024

5. Mansouri A, Hallonet M, Gruss P: Pax genes and their roles in cell differentiation and development . Curr Opin Cell Biol. 1996, 8:851-57. 10.1016/s0955-0674(96)80087-1

6. Plachov D, Chowdhury K, Walther C, Simon D, Guenet JL, Gruss P: Pax8, a murine paired box gene expressed in the developing excretory system and thyroid gland. Development. 1990, 110:643-51.

7. Tong GX, Yu WM, Beaubier NT, Weeden EM, Hamele-Bena D, Mansukhani MM, O'Toole KM: Expression of PAX8 in normal and neoplastic renal tissues: an immunohistochemical study. Mod Pathol. 2009, 22:121827. 10.1038/modpathol.2009.88

8. Nonaka D, Tang Y, Chiriboga L, Rivera M, Ghossein R: Diagnostic utility of thyroid transcription factors Pax8 and TTF-2 (FoxE1) in thyroid epithelial neoplasms. Mod Pathol. 2008, 21:192-200. 10.1038/modpathol.3801002

9. Bowen NJ, Logani S, Dickerson EB, Kapa LB, Akhtar M, Benigno BB, McDonald JF: Emerging roles for PAX8 in ovarian cancer and endosalpingeal development. Gynecol Oncol. 2007, 104:331-37. 10.1016/j.ygyno.2006.08.052

10. Laury AR, Hornick JL, Perets R, Krane JF, Corson J, Drapkin R, Hirsch MS: PAX8 reliably distinguishes ovarian serous tumors from malignant mesothelioma. Am J Surg Pathol. 2010, 34:627-35. 10.1097/PAS.0b013e3181da7687

11. Lotan TL, Ye H, Melamed J, Wu XR, Shih Ie-M, Epstein JI: Immunohistochemical panel to identify the primary site of invasive micropapillary carcinoma. Am J Surg Pathol. 2009, 33:1037-41. 10.1097/PAS.0b013e3181962dcd

12. Nonaka D, Chiriboga L, Soslow RA: Expression of pax8 as a useful marker in distinguishing ovarian carcinomas from mammary carcinomas. Am J Surg Pathol. 2008, 32:1566-71. 10.1097/PAS.0b013e31816d71ad

13. Laury AR, Perets R, Piao H, et al.: A comprehensive analysis of PAX8 expression in human epithelial tumors . Am J Surg Pathol. 2011, 35:816-26. 10.1097/PAS.0b013e318216c112

14. Ye J, Hameed O, Findeis-Hosey JJ, et al.: Diagnostic utility of PAX8, TTF-1 and napsin A for discriminating metastatic carcinoma from primary adenocarcinoma of the lung. Biotech Histochem. 2012, 87:30-34. 10.3109/10520295.2011.591838

15. Blake JA, Ziman MR: Pax genes: regulators of lineage specification and progenitor cell maintenance . Development. 2014, 141:737-51. 10.1242/dev.091785

16. Fernández LP, López-Márquez A, Santisteban P: Thyroid transcription factors in development, differentiation and disease. Nat Rev Endocrinol. 2015, 11:29-42. 10.1038/nrendo.2014.186

17. Sharma R, Sanchez-Ferras O, Bouchard M: Pax genes in renal development, disease and regeneration . Semin Cell Dev Biol. 2015, 44:97-106. 10.1016/j.semcdb.2015.09.016

18. Park SM, Chatterjee VK: Genetics of congenital hypothyroidism. J Med Genet. 2005, 42:379-89. 10.1136/jmg.2004.024158

19. Macchia PE, Lapi P, Krude H, et al.: PAX8 mutations associated with congenital hypothyroidism caused by thyroid dysgenesis. Nat Genet. 1998, 19:83-86. 10.1038/ng0598-83

20. Di Palma T, Filippone MG, Pierantoni GM, et al.: Pax8 has a critical role in epithelial cell survival and proliferation. Cell Death Dis. 2013, 4:729-29. 10.1038/cddis.2013.262

21. Xiang L, Kong B: PAX8 is a novel marker for differentiating between various types of tumor, particularly ovarian epithelial carcinomas. Oncol Lett. 2013, 5:735-38. 10.3892/ol.2013.1121

22. Placzkowski KA, Reddi HV, Grebe SK, Eberhardt NL, McIver B: The role of the PAX8/PPARY fusion oncogene in thyroid cancer. PPAR Res. 2008, 2008:672829. 10.1155/2008/672829

23. Auwerx J: PPARgamma, the ultimate thrifty gene. Diabetologia. 1999, 42:1033-49. $10.1007 \% 2 F s 001250051268$ 
24. Kroll TG, Sarraf P, Pecciarini L, Chen CJ, Mueller E, Spiegelman BM, Fletcher JA: PAX8-PPARgamma1 fusion oncogene in human thyroid carcinoma. Science. 2000, 289:1357-60. 10.1126/science.289.5483.1357

25. Bishop JA, Sharma R, Westra WH: PAX8 immunostaining of anaplastic thyroid carcinoma: a reliable means of discerning thyroid origin for undifferentiated tumors of the head and neck. Hum Pathol. 2011, 42:187377. 10.1016/j.humpath.2011.02.004

26. Roque L, Nunes VM, Ribeiro C, Martins C, Soares J: Karyotypic characterization of papillary thyroid carcinomas. Cancer. 2001, 92:2529-38. 10.1002/1097-0142(20011115)92:10<2529::aid-cncr1604>3.0.co;2-m

27. Bleu M, Gaulis S, Lopes R, et al.: PAX8 activates metabolic genes via enhancer elements in renal cell carcinoma. Nat Commun. 2019, 10:3739. 10.1038/s41467-019-11672-1

28. Barr ML, Jilaveanu LB, Camp RL, Adeniran AJ, Kluger HM, Shuch B: PAX-8 expression in renal tumours and distant sites: a useful marker of primary and metastatic renal cell carcinoma?. J Clin Pathol. 2015, 68:12-17. 10.1136/jclinpath-2014-202259

29. Hu Y, Hartmann A, Stoehr C, et al.: PAX8 is expressed in the majority of renal epithelial neoplasms: an immunohistochemical study of 223 cases using a mouse monoclonal antibody. J Clin Pathol. 2012, 65:25456. 10.1136/jclinpath-2011-200508

30. Tacha D, Zhou D, Cheng L: Expression of PAX8 in normal and neoplastic tissues: a comprehensive immunohistochemical study. Appl Immunohistochem Mol Morphol. 2011, 19:293-99. 10.1097/PAI.0b013e3182025f66

31. Ozcan A, Shen SS, Hamilton C, Anjana K, Coffey D, Krishnan B, Truong LD: PAX 8 expression in nonneoplastic tissues, primary tumors, and metastatic tumors: a comprehensive immunohistochemical study. Mod Pathol. 2011, 24:751-64. 10.1038/modpathol.2011.3

32. Knoepp SM, Kunju LP, Roh MH: Utility of PAX8 and PAX2 immunohistochemistry in the identification of renal cell carcinoma in diagnostic cytology. Diagn Cytopathol. 2012, 40:667-72. 10.1002/dc.21590

33. Shuch B, Said J, LaRochelle JC, et al.: Histologic evaluation of metastases in renal cell carcinoma with sarcomatoid transformation and its implications for systemic therapy. Cancer. 2010, 116:616-24. 10.1002/cncr.24768

34. Tan PH, Cheng L, Rioux-Leclercq N, et al.: Renal tumors: diagnostic and prognostic biomarkers . Am J Surg Pathol. 2013, 37:1518-31. 10.1097/PAS.0b013e318299f12e

35. Ozcan A, de la Roza G, Ro JY, Shen SS, Truong LD: PAX2 and PAX8 expression in primary and metastatic renal tumors: a comprehensive comparison. Arch Pathol Lab Med. 2012, 136:1541-51. 10.5858/arpa.20120072-OA

36. Mhawech-Fauceglia P, Wang D, Samrao D, Godoy H, Pejovic T, Liu S, Lele S: Pair-Box (PAX8) proteinpositive expression is associated with poor disease outcome in women with endometrial cancer. Br J Cancer. 2012, 107:370-74. 10.1038/bjc.2012.241

37. Brunner AH, Riss P, Heinze G, Meltzow E, Brustmann H: Immunoexpression of PAX 8 in endometrial cancer: relation to high-grade carcinoma and p53. Int J Gynecol Pathol. 2011, 30:569-75. 10.1097/PGP.0b013e31821ac6c3

38. Yemelyanova A, Gown AM, Wu LS, Holmes BJ, Ronnett BM, Vang R: PAX8 expression in uterine adenocarcinomas and mesonephric proliferations. Int J Gynecol Pathol. 2014, 33:492-99. 10.1097/PGP.0b013e3182a54afa

39. López-Urrutia E, Pedroza-Torres A, Fernández-Retana J, et al.: PAX8 is transcribed aberrantly in cervical tumors and derived cell lines due to complex gene rearrangements. Int J Oncol. 2016, 49:371-80. 10.3892/ijo.2016.3515

40. Li CG, Nyman JE, Braithwaite AW, Eccles MR: PAX8 promotes tumor cell growth by transcriptionally regulating E2F1 and stabilizing RB protein. Oncogene. 2011, 30:4824-34. 10.1038/onc.2011.190

41. Fu DJ, De Micheli AJ, Bidarimath M, Ellenson LH, Cosgrove BD, Flesken-Nikitin A, Nikitin AY: Cells expressing PAX8 are the main source of homeostatic regeneration of adult mouse endometrial epithelium and give rise to serous endometrial carcinoma. Dis Model Mech. 2020, 13:dmm047035. 10.1242/dmm.047035

42. Ramachandran D, Wang Y, Schürmann P, et al.: Association of genomic variants at PAX8 and PBX2 with cervical cancer risk. Int J Cancer. 2021, 149:893-900. 10.1002/ijc.33614

43. Shukla A, Thomas D, Roh MH: PAX8 and PAX2 expression in endocervical adenocarcinoma in situ and highgrade squamous dysplasia. Int J Gynecol Pathol. 2013, 32:116-21. 10.1097/PGP.0b013e318257df46

44. Maenaka T, Iwata N, Egawa-Takata T, et al.: A case of poorly differentiated adenocarcinoma of the uterine cervix treated with paclitaxel and carboplatin after positive PAX 8 immunostaining (article in Japanese). Gan To Kagaku Ryoho. 2013, 40:1413-17.

45. Tong GX, Devaraj K, Hamele-Bena D, et al.: Pax8: a marker for carcinoma of Müllerian origin in serous effusions. Diagn Cytopathol. 2011, 39:567-74. 10.1002/dc.21426

46. Woodard AH, Yu J, Dabbs DJ, et al.: NY-BR-1 and PAX8 immunoreactivity in breast, gynecologic tract, and other CK7+ carcinomas: potential use for determining site of origin. Am J Clin Pathol. 2011, 136:428-35. 10.1309/AJCPUFNMEZ3MK1BK

47. Danialan R, Assaad M, Burghardt J, Newcomb P, Cartun RW, Mandavilli S: The utility of PAX8 and IMP3 immunohistochemical stains in the differential diagnosis of benign, premalignant, and malignant endocervical glandular lesions. Gynecol Oncol. 2013, 130:383-88. 10.1016/j.ygyno.2013.04.020

48. Goyal A, Yang B: Differential patterns of PAX8, p16, and ER immunostains in mesonephric lesions and adenocarcinomas of the cervix. Int J Gynecol Pathol. 2014, 33:613-19. 10.1097/PGP.0000000000000102

49. Liang L, Zheng W, Liu J, Liang SX: Assessment of the utility of PAX8 immunohistochemical stain in diagnosing endocervical glandular lesions. Arch Pathol Lab Med. 2016, 140:148-52. 10.5858/arpa.20150081-OA

50. Gailey MP, Bellizzi AM: Immunohistochemistry for the novel markers glypican 3, PAX8, and p40 ( $\triangle$ Np63) in squamous cell and urothelial carcinoma. Am J Clin Pathol. 2013, 140:872-80. 10.1309/AJCP4NSKW5TLGTDS

51. Wong S, Hong W, Hui P, Buza N: Comprehensive analysis of PAX8 expression in epithelial malignancies of the uterine cervix. Int J Gynecol Pathol. 2017, 36:101-106. 10.1097/PGP.0000000000000309

52. Jones MW, Onisko A, Dabbs DJ, Elishaev E, Chiosea S, Bhargava R: Immunohistochemistry and HPV in situ hybridization in pathologic distinction between endocervical and endometrial adenocarcinoma: a 
comparative tissue microarray study of 76 tumors. Int J Gynecol Cancer. 2013, 23:380-84. 10.1097/IGC.0b013e31825cc8ee

53. Momenimovahed Z, Tiznobaik A, Taheri S, Salehiniya H: Ovarian cancer in the world: epidemiology and risk factors. Int J Womens Health. 2019, 11:287-99. 10.2147/IJWH.S197604

54. Hardy LR, Salvi A, Burdette JE: UnPAXing the divergent roles of PAX2 and PAX8 in high-grade serous ovarian cancer. Cancers (Basel). 2018, 10:262. 10.3390/cancers10080262

55. Di Palma T, Lucci V, de Cristofaro T, Filippone MG, Zannini M: A role for PAX8 in the tumorigenic phenotype of ovarian cancer cells. BMC Cancer. 2014, 14:292. 10.1186/1471-2407-14-292

56. Zhu W, Michael CW: WT1, monoclonal CEA, TTF1, and CA125 antibodies in the differential diagnosis of lung, breast, and ovarian adenocarcinomas in serous effusions. Diagn Cytopathol. 2007, 35:370-75. 10.1002/dc.20643

57. Chai HJ, Ren Q, Fan Q, et al.: PAX8 is a potential marker for the diagnosis of primary epithelial ovarian cancer. Oncol Lett. 2017, 14:5871-75. 10.3892/ol.2017.6949

58. Moran CA, Suster S: Neuroendocrine carcinomas (carcinoid tumor) of the thymus. A clinicopathologic analysis of 80 cases. Am J Clin Pathol. 2000, 114:100-10. 10.1309/3PDN-PMT5-EQTM-HOCD

59. Zhu S, Wang ZT, Liu WZ, Zong SX, Li BS: Invasive atypical thymic carcinoid: three case reports and literature review. Onco Targets Ther. 2016, 9:6171-76. 10.2147/OTT.S109693

60. Weissferdt A, Tang X, Wistuba II, Moran CA: Comparative immunohistochemical analysis of pulmonary and thymic neuroendocrine carcinomas using PAX8 and TTF-1. Mod Pathol. 2013, 26:1554-60. 10.1038/modpathol.2013.111

61. Weissferdt A, Moran CA: Pax8 expression in thymic epithelial neoplasms: an immunohistochemical analysis. Am J Surg Pathol. 2011, 35:1305-10. 10.1097/PAS.0b013e3182260735

62. Asirvatham JR, Esposito MJ, Bhuiya TA: Role of PAX-8, CD5, and CD117 in distinguishing thymic carcinoma from poorly differentiated lung carcinoma. Appl Immunohistochem Mol Morphol. 2014, 22:372-76. 10.1097/PAI.0b013e318297cdb5

63. Bornfeld N, Biewald E, Bauer S, Temming P, Lohmann D, Zeschnigk M: The interdisciplinary diagnosis and treatment of intraocular tumors. Dtsch Arztebl Int. 2018, 115:106-11. 10.3238/arztebl.2018.0106

64. Mudhar HS, Milman T, Eagle RC Jr, et al.: Usefulness of PAX8 immunohistochemistry in adult intraocular tumor diagnosis. Ophthalmology. 2021, 128:765-78. 10.1016/j.ophtha.2020.09.033

65. Appleford C, Zhang P: PAX-8 expression in retinoblastoma and the eyes of human embryos . Am J Clin Pathol. 2018, 150:48-49. 10.1093/ajcp/aqy090.119

66. Milman T, Mudhar HS, Eagle RC Jr: PAX8 expression in the crystalline lens and lens-derived lesions . Ophthalmol Sci. 2021, 1:100024. 10.1016/j.xops.2021.100024 